\title{
Medial temporal structures relate to memory impairment in Alzheimer's disease: an MRI volumetric study
}

Etsuro Mori, Yukihiro Yoneda, Hikari Yamashita, Nobutsugu Hirono, Manabu Ikeda, Atsushi Yamadori

\begin{abstract}
Objectives-Memory impairment is not only the earliest clinical symptom but a central and prominent feature throughout the course of Alzheimer's disease. Alzheimer related pathological alterations in the medial temporal structures may account for the memory impairments in patients with Alzheimer's disease. The aim of this study was to elucidate the role of the medial temporal structures in memory impairment caused by Alzheimer's disease.
\end{abstract}

Methods-Using high resolution MRI and a semiautomated image analysis technique, volumes of the medial temporal structures (amygdaloid complex, hippocampal formation, subiculum, and parahippocampal gyrus) were measured, and correlations between atrophy of each structure and memory dysfunction in patients with Alzheimer's disease were examined.

Results-Patients with Alzheimer's disease showed poor performance on verbal and non-verbal memory tests, and MRI volumetry showed a significant volume reduction of the medial temporal lobe structures. Volumes of the amygdaloid complex and of the subiculum correlated with memory performance. Stepwise regression analyses disclosed that the volume of the right amygdaloid complex specifically predicted visual memory function and to some extent verbal memory function, and that the volume of the left subiculum specifically predicted verbal memory function. Atrophy of the hippocampus did not predict severity of memory impairment.

Conclusions-The presence of perihippocampal damage involving the amygdala proper, its surrounding cortex, and the subiculum further increased the severity of memory impairment attributable to hippocampal damage in Alzheimer's disease.

(F Neurol Neurosurg Psychiatry 1997;63:214-221)
Neuropathological changes in Alzheimer's disease include neurofibrillary tangles, senile plaques, and neuronal and synaptic loss and
Keywords: Alzheimer's disease; magnetic resonance imaging; memory; medial temporal lobe

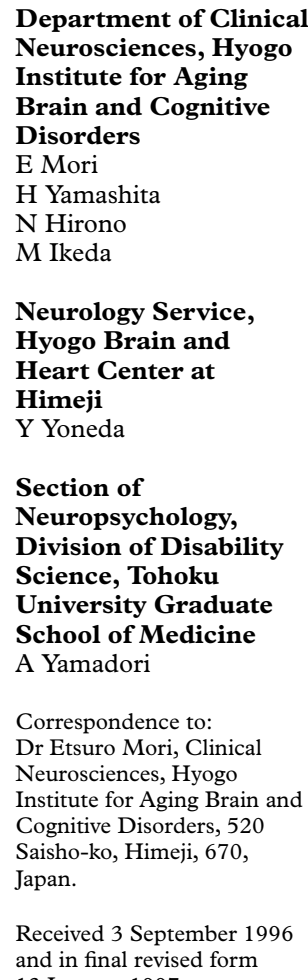

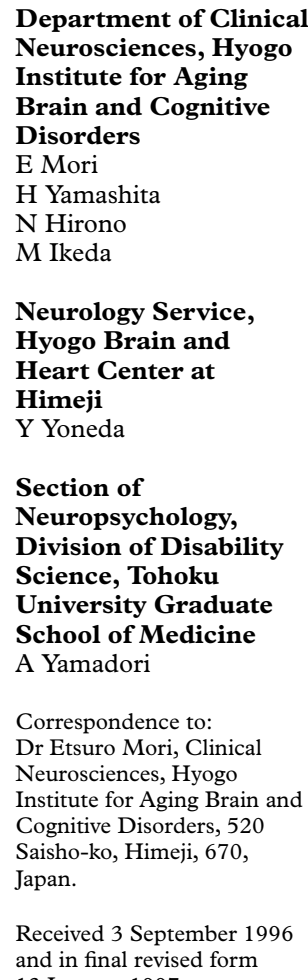

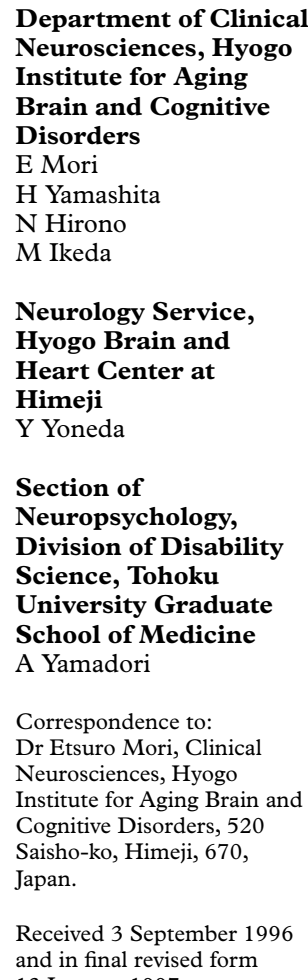

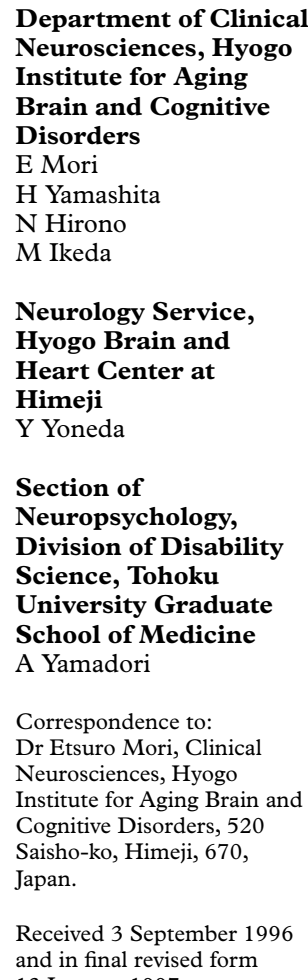

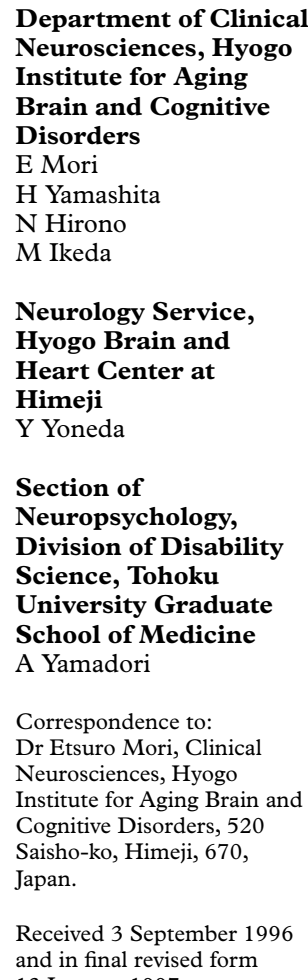

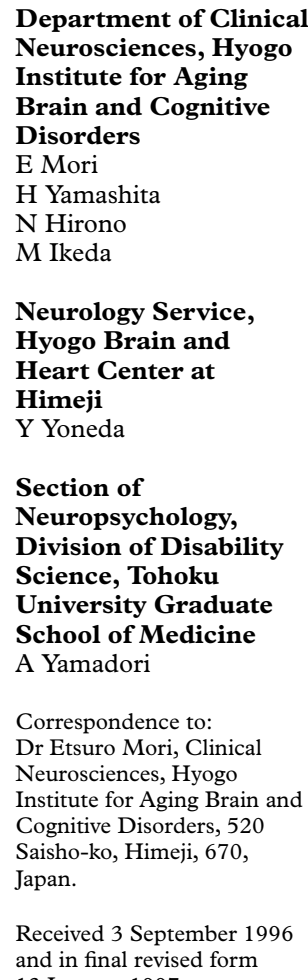

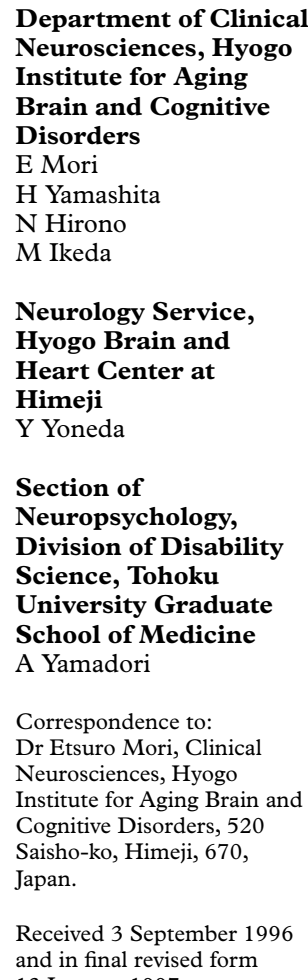

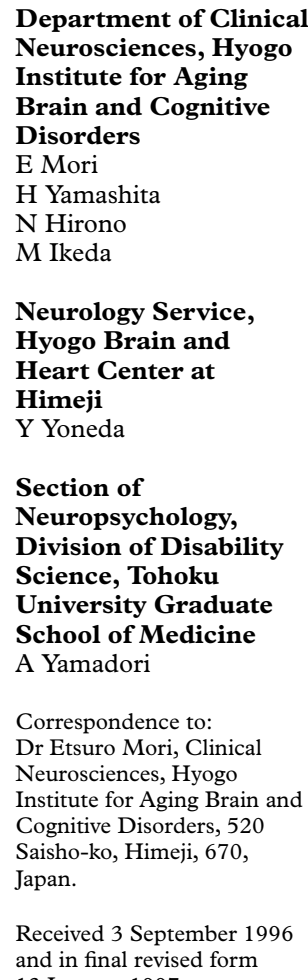

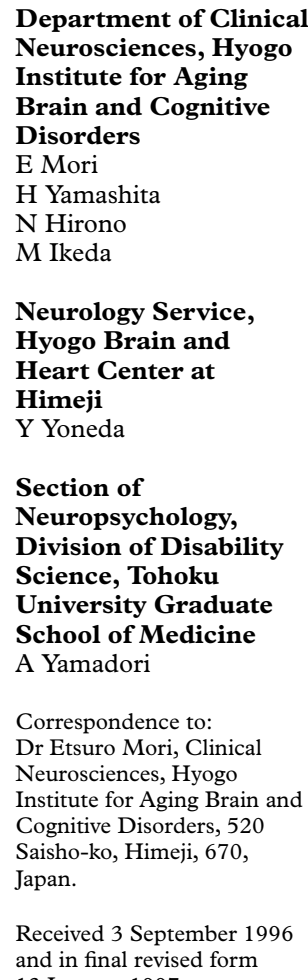 \\ 13 January 1997 \\ Accepted 6 March 1997
}

predominantly affect regions of the limbic and paralimbic medial temporal structures and association areas of the neocortex. ${ }^{1-3}$ There is a significant correlation between severity of dementia in Alzheimer's disease and these postmortem neuropathological findings. ${ }^{4-6}$ The neuropathological changes in the medial temporal lobe may account for memory impairment in Alzheimer's disease ${ }^{7}$; this is not only the earliest clinical symptom but a central and prominent feature throughout the course of the disease. In vivo neuroimaging techniques have shown a relation between memory impairment and the degree of medial temporal damage expressed as atrophy on MRI and hypoperfusion or hypometabolism shown by PET. ${ }^{8}$ The hippocampus is a central component of the medial temporal lobe memory system, and its structural integrity is necessary for declarative memory. ${ }^{9}$ The cortex adjacent to the hippocampus, including entorhinal, perirhinal, and parahippocampal cortices, is not simply a conduit for connecting the neocortex to the hippocampus, but is actively involved in storage of information from the neocortex before reaching the hippocampus itself. ${ }^{9}$ Destruction restricted to the bilateral hippocampi is known to produce memory deficits in animals ${ }^{10}$ and in humans. ${ }^{11}$ There is neuroimaging evidence for loss of hippocampal tissue in human diseases associated with memory impairments. ${ }^{12} 13$ Furthermore, the perirhinal cortex and the closely associated parahippocampal cortex provide the major source of cortical input to the hippocampal formation, ${ }^{9}$ and CA1 pyramidal cells send efferent fibres to the subiculum, entorhinal cortex, and several subcortical areas. ${ }^{14}$ Bilateral lesions limited to these areas produce severe memory impairment in monkeys. ${ }^{15}{ }^{16}$ Although the amygdala is not a part of the medial temporal lobe memory system for the processing of declarative memory, ${ }^{9}$ there is evidence indicating that lesions in the amygdala produce memory deficits in humans and animals. ${ }^{17-21}$ However, there is a controversy over whether damage to the amygdala contributes to the severity of memory loss. Recent studies have indicated that the additive effect of damage to the amygdala on hippocampal damage is not a consequence of damage to the amygdala itself but is due to involvement of the adjacent cortices (periamygdala, entorhinal, and perirhinal cortices) covering the surface of the amygdala and parahippocampal gyrus. ${ }^{9152223}$ The role of the amygdaloid complex, hippocampus, 
subiculum, and parahippocampal gyrus in memory impairment in patients with Alzheimer's disease remains unestablished.

Medial temporal atrophy is present on CT and MRI in most patients with Alzheimer's disease, and in vivo direct measurement of brain volume with MRI has emerged as the best method to quantify it. ${ }^{24-31}$ Volumetric studies have examined the relation between medial temporal lobe atrophy and memory impairments in diseases and aging. ${ }^{13}{ }^{24-36}$ In the present study, to elucidate the structural determinants of severity of impairment of memory within the medial temporal structures in patients with Alzheimer's disease, we directly measured the volume of these structures (amygdaloid complex, hippocampal formation, subiculum, and parahippocampal cortex) using semiautomated image processing of high resolution MRI, and analysed the relation between atrophy of each structure and memory dysfunction.

\section{Methods}

SUBJECTS

Forty six Japanese patients with mild to moderate Alzheimer's disease were selected among those admitted to our hospital for investigation between July 1993 and March 1995. All patients were examined by neurologists and psychiatrists. Routine laboratory tests, standard neuropsychological examinations, EEG, MRI of the brain, magnetic resonance angiography of the neck and head, and cerebral perfusion and metabolism studies by PET or single photon emission computed tomography (SPECT) were performed in all patients. The inclusion criteria comprised (1) a diagnosis of probable Alzheimer's disease according to the NINCDS/ADRDA criteria, ${ }^{37}$ (2) age younger than 80 years, and (3) mild to moderate functional impairment (grades 0.5, 1 , and 2 on the clinical dementia scale ${ }^{38}$ ). Patients were excluded if; (1) other neurological or systemic diseases were present, (2) a mini mental state examination $(\mathrm{MMSE})^{39}{ }^{40}$ score less than 11 suggested the presence of severe language, attention, and behavioural disorders that would make the neuropsychological assessment difficult, (3) evidence of focal brain lesions on MRI, and (4) inability to obtain informed consent from patients and their relatives. Twenty seven women and 19 men were selected; the mean (SD) age was 70.3 (7.1) years (range 44 to 79 ); the mean educational attainment was 8.7 (2.3) years (range 6-16); and the mean MMSE score was 19.6 (3.5) (range 12-26). All patients were right handed.

To obtain normative data for comparison, 12 age and sex matched (seven women and five men), right handed normal subjects (mean age 66.2 (4.9) years and mean educational attainment of 10.1 (2.7) years) were recruited from the community. Controls had no abnormal findings on MRI and scored 28 or more on the MMSE.

The study followed the clinical study guidelines of the ethics committee of Hyogo Institute for Aging Brain and Cognitive Disorders, Himeji Japan, and was approved by the internal review board in 1993. After a complete description of the study to the subjects and their relatives, written consent was obtained.

\section{MRI VOLUMETRY}

Brain MRI was performed on a $1.5 \mathrm{~T}$ MRI scanner (Signa Advantage 5x, General Electric) with the use of a circularly polarised head coil as both transmitter and receiver. Coronal 3D spoiled gradient echo (SPGR) images (field of view of $220 \mathrm{~mm}$, matrix of $256 \times 256,124 \times$ $1.5 \mathrm{~mm}$ contiguous sections, TR $14 \mathrm{~ms}$, TE 3 $\mathrm{ms}$, flip angle $20^{\circ}$ ) perpendicular to the anterior-posterior commissure plane covering the whole calvarium were used for the volumetric study. ${ }^{41}$ Axial T2 weighted proton density, and $\mathrm{T} 1$ weighted images were also obtained for diagnosis.

The MRI data sets of all images were directly transmitted to a personal computer (Power Macintosh 8100/80, Apple) and analysed using the public domain NIH Image version 1.59 program (written by Wayne Rasband at the US National Institutes of Health and available from the internet by anonymous ftp from zippy.nimh.nih.gov or on floppy disk from NTIS, 5285 Port Royal Rd, Springfield, VA 22161, part number PB93-504868) with residential macroprograms developed in our institute after an appropriate data conversion.

All volume measurements were performed by a segmentation technique with a combination of tracing and thresholding. The volume of a given structure in each slice was obtained by automatically counting the number of pixels within the segmented regions and multiplying the number by a voxel size $\left(220 / 256^{2} \times 1.5=\right.$ $1.108 \mathrm{~mm}^{3}$ ). One investigator (YY) measured the volume of the medial temporal structures and one (EM) the intracranial volumes. We used a semiautomatic segmentation technique through density thresholding, thereby avoiding the partial voluming and observer's bias. The test-retest reliability, measured using an intraclass correlation coefficient derived from three repeated measurements by one rater under blind conditions in 10 randomly selected subjects, was 0.990 for the intracranial volume, 0.868 for the right amygdaloid complex, 0.912 for the right hippocampal formation, 0.810 for the right subiculum, and 0.909 for the right parahippocampal cortex. In a validity experiment that used phantoms filled with copper sulphate solution, accuracies of $99.4 \%$ (average) for a $2664 \mathrm{ml}$ sphere and $98.3 \%$ (average) for a $5 \mathrm{ml}$ cylinder were achieved, indicating that measurements resulted in a slight underestimation of volume. ${ }^{41}$

For the total intracranial volume measurement, the cranial cavity was semiautomatically segmented with a density threshold set above the minimum value of the CSF in the lateral ventricle. When density thresholding resulted in insufficient segmentation, manual outlining was performed following the inner table of the skull and the basal margins of the cerebral hemispheres. ${ }^{41}$ 


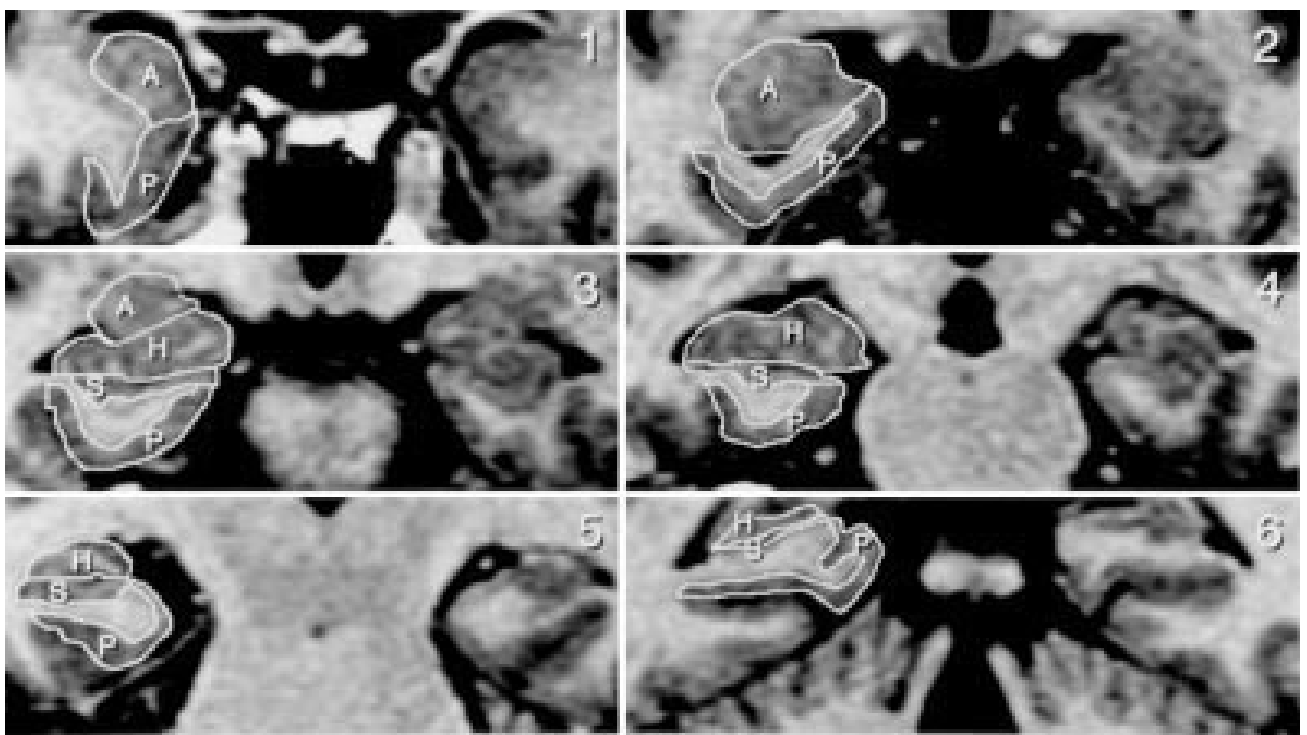

Figure 1 Boundaries of medial temporal structures on MRI. Selected slices from a 59 year old female patient with mild Alzheimer's disease and atrophic medial temporal structures for volume measurement. The most rostral slice intersects the anterior border of the amygdaloid complex (panel 1) and the most caudal slice intersects the posterior border of the hippocampal formation/parahippocampal gyrus (panel 6). A=amygdaloid complex; $H=h i p p o c a m p a l$ formation; $S=$ subiculum; $P=$ parahippocampal cortex.

Volumetry of the medial temporal structures was done with a combination of tracing and thresholding techniques. The outline of each structure including white matter and CSF was first traced with a manually driven mouse cursor, and subsequently the grey matter of each structure was extracted by density thresholding set within a range of values. The maximum value was the mean pixel value of the grey matter (represented by the caudate head), and the minimum value was the median value between the mean value of the grey matter and the mean value of the CSF (represented by the lateral ventricle).

Boundaries for the amygdaloid complex, hippocampal formation, subiculum, and parahippocampal cortex were defined in the following manner (fig 1). The amygdaloid complex was considered to include the amygdala proper (the deep/basolateral and superficial/corticomedial nuclei, and other nuclei and area), gyrus semilunaris, and the gyrus ambiens which constitutes the olfactory portion of the entorhinal cortex..$^{132}$ The amygdaloid complex is an ovoid shaped mass of grey matter. Its anterior boundary was defined as the plane including the most anterior part of the temporal stem, where the amygdaloid complex appears as a thickening of the anterior grey matter. ${ }^{28}$ Anteriorly, the amygdaloid complex was separated from the hippocampal head as a marker of the alveus hippocampi, the semianular (amygdaloid) sulcus, or the uncal recess of the inferior horn of the lateral ventricle. ${ }^{13}$ Posteriorly, a medioinferior part of the amygdaloid complex is covered by the superior medial part of the parahippocampal gyrus. At this level, the separation of the amygdaloid complex was done by drawing a line from the tentorial indentation (uncal notch) to the uncal recess of the lateral ventricle. ${ }^{13}{ }^{31}$ The inferior and lateral borders were formed by the uncal recess of the lateral ventricle or white matter, and the superior border was defined as the choroidal fissure.

The hippocampal formation was defined as comprising the pes hippocampi, digitationes hippocampi, alveus hippocampi, and dentate gyrus. ${ }^{13}$ The most posterior boundary was the section on which the crus of the fornix was seen in full profile. ${ }^{43}$ The outline boundary was manually guided along the hippocampal sulcus, medially to the inferior horn of the lateral ventricle, and turned up laterally along the choroidal fissure. ${ }^{13}{ }^{43}$ When the hippocampal digitations were fused to the amygdala, the thin line formed by the alveus served as a useful landmark.

The subiculum was defined separately from the hippocampal formation and parahippocampal gyrus. The boundary between the subiculum and hippocampal formation was the line from the hippocampal sulcus horizontally to the inferior horn of the lateral ventricle. ${ }^{13} 36$ The inferior border of the subiculum was the grey and white matter junction between the subiculum and the white matter in the parahippocampal gyrus, which was detected by density thresholding. The limit between the subiculum and parahippocampal cortex was arbitrarily defined by a line in continuation with the inferior border of the subiculum. The anteroposterior boundaries for the subiculum were similar to those for the hippocampal formation.

The parahippocampal cortex was defined as including grey matter in the parahippocampal gyrus. The separation between the amygdaloid complex and the anterior parahippocampal gyrus was mentioned above. The anterior boundary was the plane including the most anterior part of the temporal stem, and the posterior boundary for the parahippocampal gyrus was similar to that for the hippocampal formation. The internal boundary was the 
Table 1 Volumes of medial temporal structures in normal subjects and patients with Alzheimer's disease

\begin{tabular}{llll}
\hline & $\begin{array}{l}\text { Normal } \\
\text { subjects }\end{array}$ & Patients & Pvalue* \\
\hline Hippocampal formation: & & & \\
$\quad$ Right & $2411(229)$ & $2030(459)$ & 0.0002 \\
$\quad$ Left & $2218(208)$ & $1873(355)$ & $<0.001$ \\
$\begin{array}{l}\text { Subiculum: } \\
\quad \text { Right }\end{array}$ & $893(121)$ & $737(159)$ & $<0.001$ \\
$\quad$ Left & $882(117)$ & $761(148)$ & 0.004 \\
$\begin{array}{l}\text { Amygdaloid complex: } \\
\quad \text { Right }\end{array}$ & $1941(170)$ & $1572(303)$ & $<0.001$ \\
$\quad$ Left & $1761(127)$ & $1433(339)$ & $<0.001$ \\
$\begin{array}{l}\text { Parahippocampal cortex: } \\
\quad \text { Right }\end{array}$ & $1946(513)$ & $1549(561)$ & 0.002 \\
$\quad$ Left & $2237(551)$ & $1649(519)$ & $<0.001$
\end{tabular}

Results are means (SD) $\mathrm{mm}^{3}$.

*Difference between normal subjects and patients with Alzheimer's disease (two way ANOVA and post hoc Tukey test).

grey-white matter junction, which was detected by density thresholding.

NEUROPSYCHOLOGICAL ASSESSMENTS

Neuropsychological and MRI studies were performed within a one month interval. Memory functions were assessed using the following tests for verbal memory: (1) the word recall subtest of the Alzheimer's disease assessment scale (ADAS), ${ }^{44}{ }^{45}$ (2) the logical memory subtest of the Wechsler memory scale-revised (WMS-R), ${ }^{46}$ and (3) the verbal paired associates subtest of the WMS-R. For visuospatial memory examination, the following tests were used: (1) the visual paired associates subtest of the WMS-R, (2) the visual reproduction subtest of the WMS-R, and (3) the figure memory subtest of the WMS-R. In all WMS-R subtests, non-delayed recall measures were considered. The ADAS word recall subtest is equivalent to a verbal learning test, in which the retention of a written 10 word list was measured by free immediate recall after each of three learning trials. The score is expressed as the mean number of words recalled in three trials. We analysed each measure separately.

STATISTICAL ANALYSIS

To adjust for between subject variability in cranium size, the right and left volumes of the temporal lobe structures were normalised by covariance $^{42} 47$ as calculated by the following equation:

Normalised volume $=$ observed volume $-\mathrm{B}$ $\times$ (patient's total intracranial volume - mean total intracranial volume for normal subjects)

where $\mathrm{B}$ is the regression coefficient in the normative linear object volume-total intracranial volume regression. The normative relation was determined by simple regression analysis. The difference in each memory test score between the controls and patients was analysed by two tailed Student's $t$ test. Analysis of variance (ANOVA) with one between subject factor, groups (patients and normal subjects), and one within subject factor, side (left and right), was used to compare volume of each medial temporal structure, followed by Tukey's test for post hoc analysis.

The relation between the scores on each memory test and volume of each medial temporal structure in patients was explored by
Table 2 Performance of memory tests in normal subjects and patients with Alzheimer's disease

\begin{tabular}{lrrr}
\hline & \multicolumn{1}{c}{ Controls } & Patients & Pvalue \\
\hline Word recall & $7.3(0.7)$ & $3.6(1.3)$ & $<0.001$ \\
Verbal paired associates & $14.8(1.8)$ & $6.3(3.2)$ & $<0.001$ \\
Logical memory & $19.6(6.6)$ & $3.7(3.0)$ & $<0.001$ \\
Figure memory & $5.4(1.1)$ & $4.2(1.4)$ & 0.008 \\
Visual paired associates & $5.9(3.5)$ & $3.6(1.7)$ & 0.002 \\
Visual reproduction & $31.5(4.1)$ & $11.9(6.3)$ & $<0.001$
\end{tabular}

Results are means (SD).

${ }^{\star}$ Difference between normal subjects and patients (Student's $t$ test).

Pearson's correlation coefficients. Because of the inconclusive and exploratory nature of this analysis, no correction for multiple comparisons was applied. To find out the specific structural predictors of memory performance while controlling for age, sex, and education, we carried out a stepwise linear regression analysis with those confounding factors forced into the model, and with each memory test score as the dependent variable and volumes of the structures as the independent variables. The significant level was set at $\mathrm{P}<0.05$. All statistical analyses were carried out on SAS release 6.10 (SAS Institute Inc, Cary, NC, USA).

\section{Results}

BRAIN VOLUME

Table 1 summarises the volume measurements. The right and left mean volumes of the amygdaloid complex, hippocampal formation, subiculum, and parahippocampal cortex in patients with Alzheimer's disease were significantly smaller than those in normal subjects. The mean (SD) total intracranial volume was comparable between the patients with Alzheimer's disease $(1463(120) \mathrm{ml}$ ) and controls (1458 (100) ml). For patients with Alzheimer's disease, the volumes of each medial temporal structure did not correlate with age and educational attainment, and were not significantly different between women and men.

MEMORY ASSESSMENTS

The performance on memory tests was severely impaired in patients with Alzheimer's disease (table 2). Memory performance did not correlate with age and educational attainment, and no significant differences were found between women and men.

RELATION BETWEEN VOLUMES OF MEDIAL

TEMPORAL STRUCTURES AND MEMORY

PERFORMANCE

Table 3 shows the results of correlation analysis. The right and left amygdala volume and the left subicular volume were significantly correlated with memory test performance. Word recall and logical memory scores correlated with left subicular volume. Verbal paired associates score correlated with right amygdala volume, as did figure memory score with right and left amygdala volumes. Visual reproduction score correlated with the volumes of the right amygdaloid complex and of the left amygdaloid complex. A significant correlation was also found between right hippocampal volume and the visual reproduction score. 
Table 3 Correlation (Pearson correlation coefficient matrix) between performance on memory tests and volumes of medial temporal structures

\begin{tabular}{|c|c|c|c|c|c|c|c|c|}
\hline & \multicolumn{2}{|l|}{ Amygdala } & \multicolumn{2}{|c|}{ Hippocampus } & \multicolumn{2}{|c|}{ Subiculum } & \multicolumn{2}{|c|}{ Parahippocampal cortex } \\
\hline & Right & Left & Right & Left & Right & Left & Right & Left \\
\hline Word recall & 0.124 & 0.128 & 0.177 & 0.088 & 0.181 & $0.337^{\star}$ & 0.151 & 0.262 \\
\hline Verbal paired associates & $0.309^{\star}$ & 0.173 & 0.032 & 0.008 & -0.062 & -0.004 & -0.185 & -0.151 \\
\hline Logical memory & 0.169 & 0.099 & 0.086 & 0.073 & 0.264 & $0.374^{\star \star}$ & -0.121 & 0.024 \\
\hline Figure memory & $0.342^{\star}$ & $0.307^{\star}$ & 0.218 & 0.103 & 0.045 & 0.260 & -0.114 & -0.055 \\
\hline Visual paired assciates & 0.000 & 0.019 & -0.063 & -0.206 & -0.165 & -0.055 & -0.076 & -0.001 \\
\hline Visual reproduction & $0.454^{\star \star \star}$ & $0.425^{\star \star \star}$ & $0.317^{\star}$ & 0.216 & -0.068 & 0.012 & -0.019 & -0.017 \\
\hline
\end{tabular}

${ }^{\star} \mathrm{P}<0.05 ;{ }^{\star \star} \mathrm{P}<0.01 ;{ }^{\star \star \star} \mathrm{P}<0.005$

Table 4 Volumetric predictors of performance on memory tests and partial correlation coefficients in stepwise linear regression analysis

\begin{tabular}{llll}
\hline Memory test score & Predictors & Partial r & P value \\
\hline Word recall & Left subiculum & 0.318 & 0.038 \\
Logical memory & Left subiculum & 0.352 & 0.021 \\
Verbal paired associates & Right amygdala & 0.340 & 0.026 \\
Figure memory & Right amygdala & 0.338 & 0.027 \\
Visual paired associates & None & - & - \\
Visual reproduction & Right amygdala & 0.472 & 0.001
\end{tabular}

Table 4 summarises the volumetric predictors of memory test performance in the stepwise regression analysis with all the confounding variables forced into the equations. The left subicular volume predicted the scores of the word recall and of logical memory, and the right amygdala volume predicted the scores of the verbal paired associates, figure memory, and visual reproduction. Figures 2 and 3 illustrate the linear volume-test performance relations

\section{Discussion}

Before discussing the impact of medial temporal atrophy on memory impairment in Alzheimer's disease, the limitations of the present study need to be mentioned. Neurodegenerative changes in Alzheimer's disease are followed by brain atrophy, which is the main gross pathological feature of Alzheimer's disease. ${ }^{1-3}$ Pathological studies on the surgically resected hippocampus of epileptic patients have shown a high correlation between neuronal cell loss and MRI volume measurements. ${ }^{48} 49$ Studies on necropsied cases of advanced Alzheimer's disease have also shown that atrophy of the amygdala is related to Alzheimer's pathology. ${ }^{3}$ These facts give a rationale for our MRI volumetric study. The ability to separate two adja- cent brain structures is a function of the inherent spatial resolution of the images, and this is particularly important when considering slice depth as this voxel dimension is greater than the dimensions in the plane of the imaging slice. The imaging voxels must therefore be of a size that is appropriate to the object being measured, and it is particularly important for volumetric studies to use thin slices. ${ }^{36}$ The three dimensional $1.5 \mathrm{~mm}$ slice MRI we used in this study was that recommended for reliable and valid volumetry. ${ }^{36}$ Nevertheless, it is difficult to separate subdivisions within a structure even with the best technique currently available. To maintain a reliable segmentation on MRI, we had to include different anatomical structures within a single region (for example, the amygdaloid complex, which contained both the amygdala proper and its surrounding cortices). Although we made use of a stereotaxic technique based on anatomical landmarks to segment structures the outline of which cannot be delineated with contrast alone (for example, the parahippocampal cortex), individual anatomical variation may have influenced the results. We must, therefore, take these limitations into consideration when interpreting the results of this study.

The present study showed that the volumes of the medial temporal lobe structures were reduced in Alzheimer's disease. This finding is consistent with those in previous MRI volumetric studies. ${ }^{24-31} 3435$ In the correlational analysis, the volumes of the right amygdaloid complex and the left subiculum were significantly correlated with several memory scores. Although it is impossible to exclude type I error in the multiple comparisons, it is reassuring that the volumes of the same structures were repeatedly seen as significant correlates.
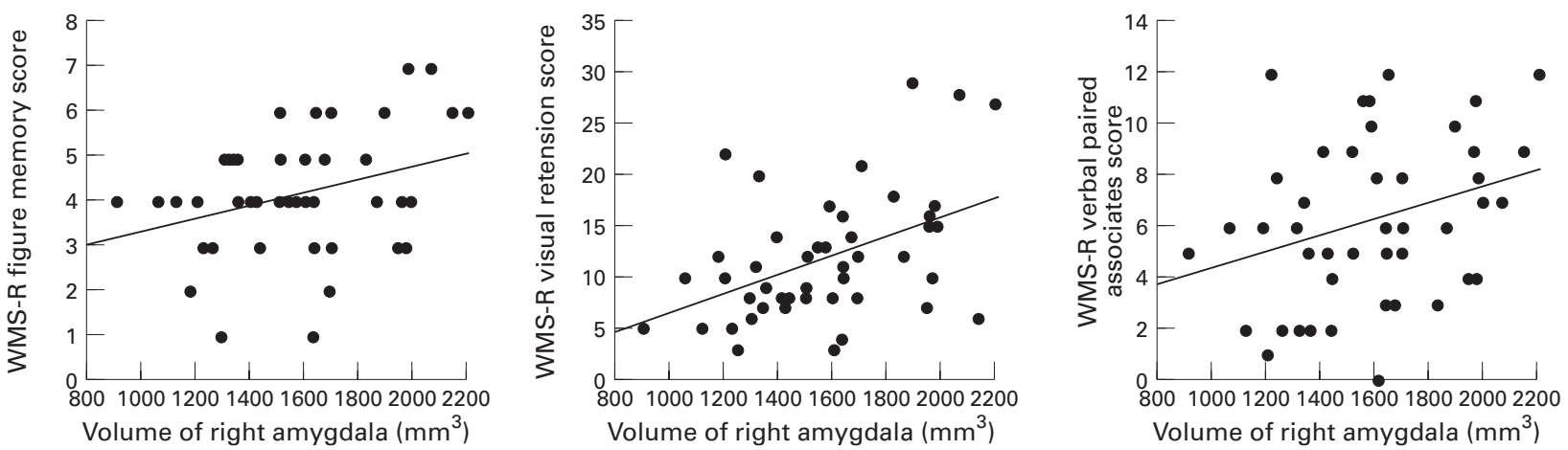

Figure 2 Linear univariate regressions of volume of the right amygdaloid complex on figure memory score $(r=0.342, P=0.020)$, on visual reproduction score $(r=0.454, P=0.002)$, and on verbal paired associates score $(r=0.309, P=0.037)$. These relations remained significant after partialling out variance due to age, sex, and education. 

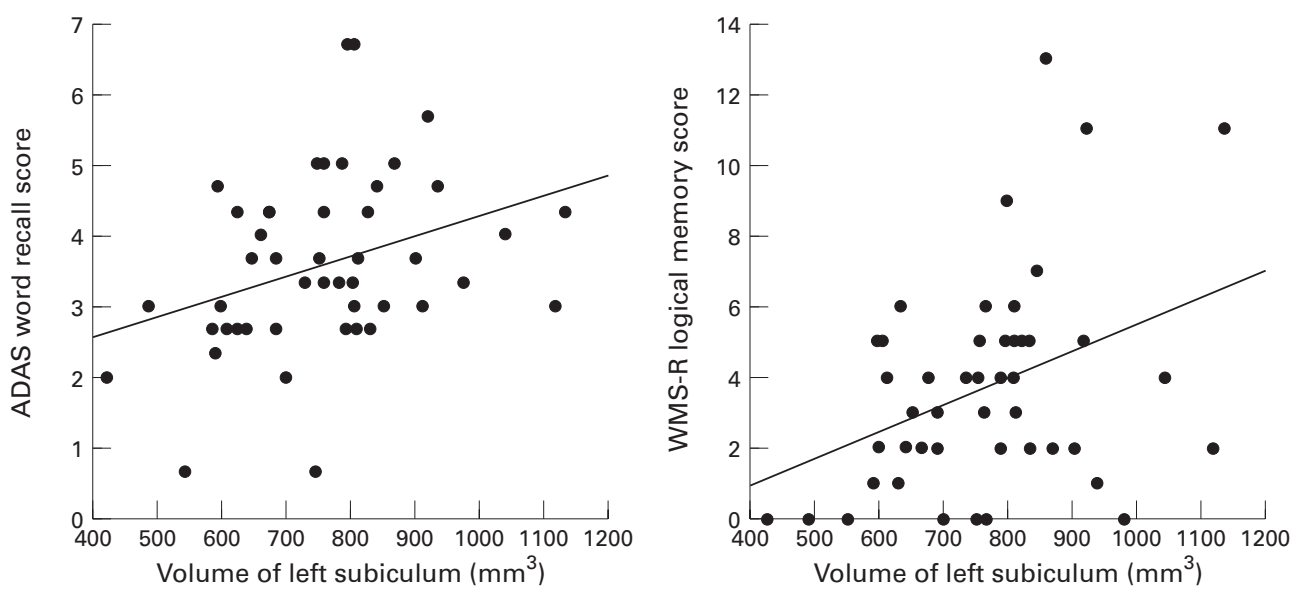

Figure 3 Linear univariate regressions of volume of the left subiculum on word recall score $(r=337, P=0.022)$ and on logical memory score $(r=0.374, P=0.010)$. These relations remained significant after partialling out variance due to age, sex, and education.

Moreover, stepwise regression analyses disclosed that, independent of age, sex, and educational attainment, the right amygdala volume specifically predicted visual and verbal memory test performance, and the left subicular volume verbal memory test performance.

Atrophy of the hippocampus was not seen as a significant predictor of severity of memory impairment. However, this does not necessarily disagree with the view of the central role of the hippocampus in the memory process. Although bilateral lesions restricted to the hippocampi produce memory impairment in animals ${ }^{910}$ and in humans, ${ }^{11}$ the deficits are limited. Our previous volumetric study in amnesic patients after herpes simplex encephalitis indicated that hippocampal damage resulting in less than $50 \%$ volume loss did not result in severe, lasting amnesia. ${ }^{13}$ As the Alzheimer's disease pathology invariably affects the hippocampal formation, damage to the hippocampus might be an indispensable condition for occurrence of memory deficits in patients with Alzheimer's disease. The damage to the amygdala proper, its surrounding cortices, and the subiculum exacerbates further the memory impairment after hippocampal damage.

Our results indicate the involvement of the left subiculum in verbal memory. In previous studies, Laakso et $a \beta^{35}$ found a significant correlation between hippocampal volume and verbal memory performance in 32 patients with Alzheimer's disease, and Deweer et $a{ }^{34}$ also noted significant correlations between verbal memory variables and hippocampal volume in a study with 18 patients with Alzheimer's disease. However, in both studies, the hippocampal volumetry included the subiculum, which is a main locus of involvement in Alzheimer's disease pathology. The association of the hippocampal volume with memory test performance shown in those studies could therefore be explained by subicular atrophy. The subiculum is one of the few major output zones for the hippocampus projecting to numerous other brain areas, and is one of the major sources of cortical input to the hippocampal formation. ${ }^{912}$ Alzheimer's disease pathology affects the subiculum and CA1 zone of the hippocampus as well as layers II and IV of the entorhinal cortex, and this focal pattern of pathology is likely to isolate the hippocampal formation from much of its input and output and probably contributes to the memory disorder in Alzheimer's disease. ${ }^{1750}$ Our findings support this view, and suggest that the subiculum is a more critical site for memory impairment in Alzheimer's disease than the hippocampus proper. The dominance of the left side in verbal memory is in agreement with many previous studies reporting verbal material specific memory deficits in patients with the left medial temporal lobe lesions including unilateral temporal lobectomy. ${ }^{51}$

On the other hand, we found a significant involvement of the right amygdaloid complex in memory impairment in Alzheimer's disease. Soininen et $a l^{33}$ in a study of age associated memory impairment, showed significant correlations of the amygdala volumes with the performance on visual memory tests but not with performance on the verbal memory tests. Whereas two previous volumetric studies on Alzheimer's disease failed to show each correlation. ${ }^{34}{ }^{35}$ Deweer et al, ${ }^{34}$ using $5 \mathrm{~mm}$ slice thick MRI, inadequate for volumetry of the amygdala, also failed to find an association between the volume of the amygdala and memory function. Laakso $e t a l^{35}$ failed to find a significant difference in the volume of the amygdala between patients with Alzheimer's disease and age matched controls, and no mention was made in that study of the correlation between volume of the amygdala and memory performance.

There are many controversies about the role of the amygdala in memory function; whether lesions restricted in the amygdala can cause memory deficits in humans and animals and whether additional lesions in the amygdala exacerbate the memory impairment caused by hippocampal lesions. The amygdala and hippocampal formation are anatomically interconnected, several amygdaloid nuclei contribute projections to the hippocampus, subiculum, and entorhinal cortex, and receive hippocampal innervation. ${ }^{52}$ An active role for the amygdala in human memory is supported by 
the studies of patients with bilateral damage to the amygdala due to Urbach-Wiethe disease, in particular a significant defect in visual memory has been reported. ${ }^{18}{ }^{19}$ Mishkin $^{20}$ discussed the discrepancy between animal and human amnesia; the profound anterograde amnesia attributed to damage of the hippocampus in human patients has not been reproduced in animals with such lesions. He showed that combined but not separate removal of the amygdala and hippocampus impaired memory in monkeys, and she stressed the importance of damage to both the hippocampus and amygdala in severe memory impairment. Based on their studies of rhesus monkeys, Murray and Gaffan ${ }^{21}$ found that the amygdala, its underlying cortices, or both, play a part in crossmodal and intramodal associative memory in animals. It has been suggested that the amygdala plays a part in integrating the memory modulating effects of systems activated by learning experiences in rodents. ${ }^{53}$ However, Zola-Morgan and his coworkers, based on a series of studies on monkeys with discrete lesions in the medial temporal lobe, have suggested that damage to the cortical regions surrounding the amygdala contributes to memory impairment. In their experiments, circumscribed bilateral lesions of the amygdala sparing the surrounding cortex (the periamygdaloid, entorhinal, and perirhinal cortices) did neither impair performance on memory tasks nor exacerbate the memory impairment that followed isolated hippocampal lesions, ${ }^{15}$ whereas the addition of lesions in the anterior entorhinal cortex and perirhinal cortex, sparing amygdala, exacerbated the memory deficit produced by the lesions in the hippocampus and its adjacent cortex..$^{23}$ They also showed that lesions of perirhinal and parahippocampal cortices that spared the amygdala and hippocampal formation produced severe memory impairment. ${ }^{22}$ Because in our study we measured both the amygdala proper and its adjacent cortical regions together, the crucial lesion producing severe amnesia remains to be determined. Further studies with more refined techniques are necessary to resolve this issue.

The present study indicates that the right amygdaloid complex was more critically involved in memory than the left. Experiments with rats have suggested that the right amygdala contributes more to the expression of memory, ${ }^{54}{ }^{55}$ although it is not always possible to extrapolate findings obtained in animals to humans. The functional lateralisation of the amygdaloid complex in humans needs to be investigated further.

We conclude that perihippocampal damage, involving the amygdala proper, its surrounding cortices, and the subiculum, added to the memory impairment caused by hippcampal damage in Alzheimer's disease. Our results also support the view that these structures play an active part in human memory.

This study was supported by a grant from the Ministry of Health and Welfare, Japan. We thank our colleagues, Drs Toru Health and Welfare, Japan. We thank our colleagues, Drs Toru
Imamura, Yoshitaka Ikejiri, and Tatsuo Shimomura, Department of Clinical Neuroscience, and Hajime Kitagaki, Department of Neuroimaging Research, for their help in various parts ment of Neuroimaging Research, for their help in various parts
of the study, and Kazuhiro Toso, Medical Informatics and
Misato Fujimori, Neuropsychology unit, for their technical assistance.

1 Hyman BT, Van Hoesen GW, Damasio AR. Memoryrelated neural systems in Alzheimer's disease: an anatomic related neural systems in Alzheimer's

2 Mann DM. The topographic distribution of brain atrophy in Alzheimer's disease. Acta Neuropathol (Berl) 1991;83: $81-6$

3 Scott SA, DeKosky ST, Scheff SW. Volumetric atrophy of the amygdala in Alzheimer's disease: quantitative serial reconstruction. Neurology 1991;41:351-6.

4 Wilcock GK, Esiri MM. Plaque, tangles and dementia: a quantitative study. F Neurol Sci 1982;56:343-56.

5 Neary D, Snowden JS, Mann DMA, et al. Alzheimer's disease: a correlative study. F Neurol Neurosurg Psychiatry 1986;49:229-37.

6 Terry RD, Masliah E, Salmon DP, et al. Physical basis of cognitive alterations in Alzheimer's disease: synapse loss is the major correlate of cognitive impairment. Ann Neurol 1991;30:572-80.

7 Hyman BT, Van Horsen GW, Damasio AR, Barnes CL. Alzheimer's disease: cell-specific pathology isolates the hippocampal formation. Science 1984;225:1168-70.

8 Ishii K, Kitagaki H, Kono M, Mori E. Decreased medial temporal oxygen metabolism in Alzheimer's disease shown by positron emission tomography. F Nucl Med 1996;37: $1159-65$.

9 Squire LR, Zola-Morgan S. The medial temporal lobe memory system. Science 1991;20:1380-6.

10 Alvarez P, Zola-Morgan S, Squire LR. Damage limited to the hippocampal region produces long-lasting memory

11 Zola-Morgan S, Squire LR, Amaral DG. Human amnesia and the medial temporal region: enduring memory impairment following a bilateral lesion limited to

12 Press GA, Amaral DG, Squire LR. Hippocampal abnormalities in amnestic patients revealed by high-resolution magnetic resonance imaging. Nature 1989;341:54-7.

13 Yoneda Y, Mori E, Yamadori A, Yamashita H. MRI volumetry of medial temporal lobe structures in amnesia following herpes simplex encephalitis. Eur Neurol 1994;34: 243-52.

14 Knowles WD. Normal anatomy and neurophysiology of the hippocampal formation. f Clin Neurophysiol 1992;9:252-

15 Zola-Morgan S, Squire LR, Amaral DG. Lesions of the amygdala that spare adjucent cortical regions do not impair memory or exacerbate the impairment following lesions of the hippocampal formation. F Neurosci 1989;9:1922-36.

16 Suzuki WA, Zola-Morgan S, Squire LR, Amaral DG. Lesions of the perirhinal and parahippocampal cortices in the monkey produce long-lasting memory impairment in the visual and tactual modalities. F Neurosci 1993;13:243051

17 Aggleton JP. The effects of amygdala lesions in humans: a comparison with findings from monkeys. In: Aggleton JP ed. The amygdala: neurobiological aspects of emotion, memory,
and mental dysfunction. New York: Wiley-Liss, 1992:485503.

18 Tranel D, Hyman BT. Neuropsychological correlates of bilateral amygdala damage. Arch Neurol 1990;47:349-55.

19 Markowitsh HJ, Calabrese P, Würker M, et al. The amygdala's contribution to memory: a study on two patients with la's contribution to memory: a study on two patients
Urbach-Wiethe disease. Neuroreport 1994;5:1349-52.

20 Mishkin M. Memory in monkeys is severely impaired by combined but not by separate removal of amygdala and combined but not by separate remov

21 Murray EA, Gaffan D. Removal of the amygdala plus adjucent cortex disrupts the retention of both intramodal and crossmodal associative memories in monkeys. Behav Neurosci 1994;108:494-500.

22 Zola-Morgan S, Squire LR, Amaral DG, Suzuki WA. Lesions of perirhinal and parahippocampal cortex that spare the amygdala and hippocampal formation produce severe memory impairment. F Neurosci 1989;9:4355-70.

23 Zola-Morgan S, Squire LR, Clower RP, Rempel NL. Damage to the perirhinal cortex exacerbates memory impairment following lesions to the hippocampal formation. $f$ Neurosci 1993;13:251-65.

24 Kesslack JP, Nalcioglu O, Cotman CW. Quantification of magnetic resonance scans for hippocampal and parahip41:51-4.

25 Jack CR Jr, Petersen RC, O'Brien PC, Tangalos EG. MR-based hippocampal volumetry in the diagnosis of Alzheimer's disease. Neurology 1992;42:183-8.

26 Pearlson GD, Harris GJ, Powers RE, et al. Quantitative changes in mesial temporal volume, regional cerebral blood flow, and cognition in Alzheimer's disease. Arch Gen Psychiatry 1992;49:402-8.

27 Erkinjuntti T, Lee DH, Gao F, et al. Temporal lobe atrophy on magnetic resonance imaging in the diagnosis of early Alzheimer's disease. Arch Neurol 1993;50:305-10.

28 Cuénod C-A, Denys A, Michot J-L, et al. Amygdala atrophy in Alzheimer's disease: an in vivo magnetic resonance imaging study. Arch Neurol 1993;50:941-5.

29 Killiany RJ, Moss MB, Albert MS, et al. Temporal lobe regions on magnetic resonance imaging identify patients with early Alzheimer's disease. Arch Neurol 1993;50:949 54 . 
30 Convit A, de Leon MJ, Golomb J, et al. Hippocampal atrophy in early Alzheimer's disease, anatomic specificity and phy in early Alzheimer's disease, anato
validation. Psychiatr $Q$ 1993;64:371-87.

31 Lehéricy S, Baulac M, Chiras J, et al. Amygdalohippocampal MR volume measurement in the early stages of Alzheimer disease. AfNR Am f Neuroradiol 1994;15:927-37.

32 Golomb J, Kluger A, de Leon M, et al. Hippocampal formation size in normal human aging: a correlate of delayed secondary memory performance. Learning Memory 1994;1: 45-54.

33 Soininen HS, Partanen K, Pitkänen A, et al. Volumetric MRI analysis of the amygdala and hippocampus in subjects with age-associated memory impairment: Correlation to visual and verbal memory. Neurology 1994;44:1660-8.

34 Deweer B, Lehéricy S, Pillon B, et al. Memory disorders in probable Alzheimer's disease: the role of hippocampal atrophy as shown with MRI. F Neurl Neurosurg Psychiatry 1995 ; $\mathbf{5 8 : 5 9 0 - 7}$

35 Laakso MP, Soininen H, Partanen K, et al. Volumes of hippocampus, amygdala and frontal lobes in the MRI-based pocampus, amygdala and frontal lobes in the MRI-based diagnosis of early Alzheimer's disease: correlation with
memory functions. $\mathcal{F}$ Neural Transm Park Dis Dement Sect memory function.

36 Jack CR Jr. MRI-based hippocampal volume measurements in epilepsy. Epilepsia 1994;35(suppl 6):21-9.

37 McKhann G, Drachman D, Folstein M, et al. Clinical diag nosis of Alzheimer's disease: report of the NINCDSADRDA Work Group under the auspices of Department of Health and Human Services Task Force on Alzheimer's disease. Neurology 1984;34:939-44.

38 Morris JC. The clinical dementia rating (CDR): current version and scoring rules. Neurology 1993;43:2412-4.

39 Folstein M F, Folstein SE, McHugh PR. Mini-mental state: a practical method for grading the cognitive state for the clinician. F Psychiatr Res 1975;12:189-98.

40 Mori E, Mitani Y, Yamadori A. Usefulness of Japanese version of the mini-mental state test in neurological patients. fapanese fournal of Neuropsychology 1985;1:82-90.

41 Mori E, Hirono N, Yamashita H, et al. Premorbid brain size as a determinant of reserve capacity against intellectual as a determinant of reserve capacity against intellectual decline

42 Jack CR, Twomey CK, Zinsmeister AR, et al. Anterior temporal lobes and hippocampal formations: normative volumetric measurements from MR images in young adults. Radiology 1989;172:549-54
43 Watson C, Andermann F, Gloor P, et al. Anatomic basis of amygdaloid and hippocampal volume measurement by magnetic resonance imaging. Neurology 1992;42:1743-50.

44 Mohs RC, Rosen WG, Davis KL. The Alzheimer's disease assessment scale: an instrument for assessing treatment efficacy. Psychopharmacol Bull 1983;19:448-50.

45 Honma A, Fukuzawa K, Tsukada Y, et al. Development of a Japanese version of Alzheimer's disease assessment scale (ADAS). Fapanese fournal of Geriatric Psychiatry 1992;3: 647-55.

46 Wechsler D. Wechsler memory scale - revised. San Antonio: Psychological Corporation, 1981.

47 Free SL, Bergin PS, Gish DR, et al. Methods for normalization of hippocampal volumes measured with MR. AfNR Am $\mathcal{F}$ Neuroradiol 1995;16:637-43.

48 Cascino GD, Jack CR Jr, Parisi JE, et al. Magnetic resonance imaging-based volume studies in temporal lobe epilepsy: pathological correlations. Ann Neurol 1991;30:31-6.

49 Lencz T, McCarthy G, Bronen RA, et al. Quantitative magnetic resonance imaging in temporal lobe epilepsy: relationship to neuropathology and neuropsychological function. Ann Neurol 1992;31:629-37.

50 Van Hoesen GW, Hyman BT. Hippocampal formation: anatomy and the patterns of pathology in Alzheimer's disease. Prog Brain Res 1990;83:445-7.

51 Smith ML. Memory disorders associated with temporallobe lesions. In: Boller F, Grafman J eds. Handbook of neuropsychology. Vol 3. Amsterdam: Elsevier, 1989:91-106.

52 Amaral DG, Price JL, Pitkänen A, Carmichael ST. Anatomical organization of the primate amygdaloid Anatomical organization of the primate amygdaloid
complex. In: Aggleton JP eds. The amygdala: neurobiological aspects of emotion, memory, and mental dysfunction. New aspects of emotion, memory, and

53 McGaugh JL, Introini-Collison IB, Cahill LF, et al. Neuromodulatory systems and memory storage: role of the amygdala. Behav Brain Res 1993;58:81-90.

54 Coleman-Mesches K, McGaugh JL. Differential involvement of the right and left amygdalae in expression of memory for aversively motivated training. Brain Res 1995; 670:75-81.

55 Coleman-Mesches K, McGaugh JL. Muscimol injected into the right or left amygdaloid complex differentially affects retention performance following aversively motivated training. Brain Res 1995;676:183-8. 